\title{
Combined Chiral Dynamics and MIT Bag Model Study of Strong $\Sigma_{Q}^{(*)} \rightarrow \Lambda_{Q} \pi$ Decays
}

\author{
Chien-Wen Hwang* \\ Department of Physics, National Kaohsiung Normal University, \\ Kaohsiung, Taiwan 824, Republic of China
}

\begin{abstract}
The strong decays of the heavy baryons $\Sigma_{Q}^{(*)} \rightarrow \Lambda_{Q} \pi$ are studied by combining the chiral dynamics and the MIT bag model. In charm sector, we calculate the decay widths $\Gamma\left(\Sigma_{c}^{(*)} \rightarrow \Lambda_{c} \pi\right)$ and compare with the experimental data and other theoretical estimations. In addition, we also predict the strong decay widths $\Gamma\left(\Sigma_{b}^{(*)} \rightarrow \Lambda_{b} \pi\right)$.
\end{abstract}

\footnotetext{
* Email: t2732@nknucc.nknu.edu.tw
} 


\section{INTRODUCTION}

Two exotic relatives of the proton and neutron $\Sigma_{b}^{(*)+}(b u u)$ and $\Sigma_{b}^{(*)-}(b d d)$ were discovered by CDF collaboration at Fermilab [1]. Their masses are: $M_{\Sigma_{b}^{+}}=5808_{-2.3}^{+2.0} \pm 1.7 \mathrm{MeV}$, $M_{\Sigma_{b}^{-}}=5816_{-1.0}^{+1.0} \pm 1.7 \mathrm{MeV}, M_{\Sigma_{b}^{*+}}=5829_{-1.8}^{+1.6} \pm 1.7 \mathrm{MeV}$, and $M_{\Sigma_{b}^{*-}}=5837_{-1.9}^{+2.1} \pm 1.7$ $\mathrm{MeV}$. Due to the mass of bottomed baryon $\Lambda_{b}^{0}$ is $5624 \pm 9 \mathrm{MeV}$ [2], and the mass differences $M_{\Sigma_{b}^{(*)}}-M_{\Lambda_{b}}$ are all larger than $M_{\pi}$, thus the dominate decay modes of these exotic bottomed baryons are the strong decays $\Sigma_{b}^{(*)} \rightarrow \Lambda_{b} \pi$, which are similar to the ones of charmed baryons $\Sigma_{c}^{(*)} \rightarrow \Lambda_{c} \pi$. In this paper, we will combine the chiral dynamics and MIT bag model to study the strong decays of the heavy baryon $\Sigma_{Q}^{(*)} \rightarrow \Lambda_{Q} \pi$.

The light quark contribution to the QCD Lagrangian

$$
\mathcal{L}_{q}=\bar{q}\left(i \not D-m_{q}\right) q, \quad(q=u, d, \text { and } s)
$$

has an approximate $\mathrm{SU}(3)_{L} \times \mathrm{SU}(3)_{R}$ flavor chiral symmetry, because the current quark masses are all very small on the typical hadron energy scale. It was known early on that this symmetry must be spontaneously broken by the QCD vacuum, so that the physical spectra of hadrons made up of light quarks have only $\mathrm{SU}(3)_{L+R}$ symmetry. Moreover, due to the spontaneous breaking of the chiral symmetry, there exist eight pseudoscalar bosons (called Goldstone bosons, which include three $\pi$ 's, four $K$ 's, and one $\eta$ ). Their couplings to hadrons at low energies are determined by partial conservation of axial-vector current (PCAC) and current algebra. The chiral properties of a heavy hadron is dictated by its light quark contents. For the heavy baryon, a heavy quark $Q$ will combine the two light quarks to form baryons $Q q_{1} q_{2}$. However, the two light quarks can form a symmetric sextet or an antisymmetric antitriplet in flavor $\mathrm{SU}(3)$ space. For the ground state baryons, the $\mathrm{SU}(3)$ symmetric sextet diquarks have spin 1 whereas the SU(3) antisymmetric antitriplet diquarks

have spin 0 . Thus for the $\mathrm{SU}(3)$ symmetric sextet there are both spin $\frac{1}{2}$ baryons $\left(B_{6}\right)$ and spin $\frac{3}{2}$ baryons $\left(B_{6}^{*}\right)$. For the $\mathrm{SU}(3)$ antisymmetric antitriplet there is only spin $\frac{1}{2}$ baryons $\left(B_{\overline{3}}\right)$. Once the flavor $\mathrm{SU}(3)$ contents of these heavy baryons are determined, their couplings to Goldstone bosons can be immediately written down following the standard formalism of chiral dynamics.

The MIT bag model [3, 4] is a simple relativistic model of hadrons which is consistent with some of the essential features of QCD, namely confinement, gluon degree of freedom, and gauge invariance. It has been applied to describe hadron spectroscopy [5, 6] and various 
transitions where momentum transfers are not large [6]. The greatest success of the MIT bag model lies in its description of ground state hadrons formed with light quarks, where agreement with the experimental data is remarkable.

The paper is organized as follows. In Sec. II we review the basic MIT bag model formalism and construct the heavy baryon wave functions. In Sec. III we consider the dynamics of heavy baryons interacting with the Goldstone bosons. We discuss the chiral properties of the heavy baryons and derive the chiral Lagrangian involving heavy baryons. Applications and numerical results for the strong decay widths of some heavy baryons are worked out in Sec. IV. Finally, the conclusion is given in Sec. V.

\section{FORMALISM OF MIT BAG MODEL FOR HEAVY BARYON}

The MIT bag model is essentially a relativistic shell model which describes quarks moving independently inside a confining spherical cavity of radius $R$. The bag Lagrangian for quark only is

$$
\mathcal{L}_{\text {bag }}=\left\{\frac{i}{2}\left[\bar{\psi} \gamma^{\mu} \partial_{\mu} \psi-\left(\partial_{\mu} \bar{\psi}\right) \gamma^{\mu} \psi\right]-m \bar{\psi} \psi-\mathcal{B}\right\} \theta_{V}(r)-\frac{1}{2} \bar{\psi} \psi \Delta_{s}(r),
$$

where $m$ is the quark mass, $\theta_{V}(r)=\theta(R-r), \Delta_{s}(r)=\delta(R-r)$, the constant $\mathcal{B}$ is the volume energy density, which comes from the work done against the QCD vacuum in creating the cavity, finally the surface term is added to $\mathcal{L}_{b a g}$ so that the quarks move as if they had an infinite mass outside the bag [7].

The Euler-Lagrange equations of motion are

$$
\left.\begin{array}{c}
\left(i \gamma^{\mu} \partial_{\mu}-m\right) \psi=0 \\
i \gamma^{\mu} n_{\mu} \psi=\psi \\
n_{\mu} \cdot \partial^{\mu}(\bar{\psi} \psi)=2 \mathcal{B}
\end{array}\right\} \quad \begin{aligned}
& \text { inside the bag } \\
& \text { on the bag surface }
\end{aligned}
$$

where $n_{\mu}$ is a covariant inward vector normal to the bag surface, $n_{\mu}=(0,-\hat{r})$ for a static spherical bag. From Eq. (3), we see that the Lagrangian density Eq. (2) yields a free Dirac equation inside the bag as expected. The quadratic boundary condition (the second line of Eq. (41)) requires that only $j=1 / 2$ modes can be excited within a static rigid bag. Consequently only $S$ and $P(l=0,1)$ orbital angular momenta are allowed, and the single particle excitation spectrum consists of two classes of states with total angular momentum $j=1 / 2$, namely $n S_{1 / 2}$ and $n P_{1 / 2}(n=$ radial quantum number $)$. Explicit expressions for 
these solutions are [6]

$$
u_{n}^{0}=N_{n}^{0}\left(\begin{array}{c}
\sqrt{\left(\frac{\omega+m}{\omega}\right)} i j_{0}\left(X_{n} \frac{r}{R}\right) \chi \\
-\sqrt{\left(\frac{\omega-m}{\omega}\right)} j_{1}\left(X_{n} \frac{r}{R}\right) \sigma \cdot \widehat{r} \chi
\end{array}\right) e^{-i \omega t}
$$

and

$$
u_{n}^{1}=N_{n}^{1}\left(\begin{array}{c}
\sqrt{\left(\frac{\omega+m}{\omega}\right)} i j_{1}\left(X_{n} \frac{r}{R}\right) \sigma \cdot \widehat{r} \chi \\
\sqrt{\left(\frac{\omega-m}{\omega}\right)} j_{0}\left(X_{n} \frac{r}{R}\right) \chi
\end{array}\right) e^{-i \omega t}
$$

where the normalization coefficients $N_{n}^{0}, N_{n}^{1}$ are fixed by the integral $\int_{R} \psi^{\dagger} \psi d^{3} r=1, j_{0}, j_{1}$ are spherical Bessel functions

$$
j_{0}(x)=\frac{\sin x}{x}, \quad j_{1}(x)=\frac{\sin x}{x^{2}}-\frac{\cos x}{x}
$$

$\chi$ is a Pauli spinor, $X_{n}$ is the quark's momentum times $R$, and $\omega$ is the eigenenergy given by

$$
\omega=\sqrt{\left(m^{2}+\frac{X_{n}^{2}}{R^{2}}\right)}
$$

The eigenvalue equations can be derived by substituting Eqs. (5) and (6) into the first line of Eq. (4). For $u_{n}^{0}$, we get

$$
\left(\begin{array}{cc}
0 & -i \sigma \cdot \widehat{r} \\
i \sigma \cdot \widehat{r} & 0
\end{array}\right)\left(\begin{array}{c}
\sqrt{\left(\frac{\omega+m}{\omega}\right)} i j_{0}\left(X_{n}\right) \\
-\sqrt{\left(\frac{\omega-m}{\omega}\right)} j_{1}\left(X_{n}\right) \sigma \cdot \widehat{r}
\end{array}\right)=\left(\begin{array}{c}
\sqrt{\left(\frac{\omega+m}{\omega}\right)} i j_{0}\left(X_{n}\right) \\
-\sqrt{\left(\frac{\omega-m}{\omega}\right)} j_{1}\left(X_{n}\right) \sigma \cdot \widehat{r}
\end{array}\right)
$$

which leads to

$$
j_{1}\left(X_{n}\right)=\sqrt{\left(\frac{\omega+m}{\omega-m}\right)} j_{0}\left(X_{n}\right)
$$

and finally

$$
\tan X_{n}=\frac{X_{n}}{1-m R-\sqrt{m^{2} R^{2}+X_{n}^{2}}} .
$$

The corresponding eigenvalue equation for the $u_{n}^{1}$ mode is

$$
\tan X_{n}=\frac{X_{n}}{1-m R+\sqrt{m^{2} R^{2}+X_{n}^{2}}} .
$$

When $m \rightarrow 0$, Eqs. (11) and (12) yield the transcendental equations

$$
\tan X_{n}=\frac{X_{n}}{1 \mp X_{n}} .
$$




\begin{tabular}{|c||c|c|c|c|c|c|}
\hline state & $1 S_{1 / 2}$ & $1 P_{1 / 2}$ & $2 S_{1 / 2}$ & $2 P_{1 / 2}$ & $3 S_{1 / 2}$ & $3 P_{1 / 2}$ \\
\hline$X_{n}$ & 2.043 & 3.812 & 5.396 & 7.002 & 8.578 & 10.163 \\
\hline
\end{tabular}

TABLE I: Bag model eigenmodes for a massless quark.

Some of the low lying solutions to Eq. (13) are listed in TABLE I [8].

After solving the bag equations of motion inside a cavity, we can expand a second quantized light quark field operator $\psi_{q}(x)$ in terms of the quark eigenmodes,

$$
\psi_{q}(x)=\sum_{n l m} b_{n l m} u_{n l m}(\vec{r}, t)+d_{n l m}^{\dagger} v_{n l m}(\vec{r}, t)
$$

where $b_{n l m}$ is the canonical quark annihilation operator and $d_{n l m}^{\dagger}$ is the antiquark creation operator. These operators satisfy the usual anticommutation relations, namely

$$
\left\{b_{n l m}, b_{n^{\prime} l^{\prime} m^{\prime}}^{\dagger}\right\}=\left\{d_{n l m}, d_{n^{\prime} l^{\prime} m^{\prime}}^{\dagger}\right\}=\delta_{n n^{\prime}} \delta_{l l^{\prime}} \delta_{m m^{\prime}}
$$

with the others equal to zero. We can then readily construct a state of heavy baryon with spin $S$ as

$$
\left|B_{Q}^{n l}\right\rangle=C\left(S, S_{z}, s_{1}, s_{2}, s_{3}\right)\left|q_{1}^{n l}\left(s_{1}\right) q_{2}^{n l}\left(s_{2}\right) Q\left(s_{3}\right)\right\rangle
$$

where $C\left(S, S_{z}, s_{1}, s_{2}, s_{3}\right)$ is the familiar Clebsch-Gordan coefficient. Each light quark is in a triplet

$$
q=\left(\begin{array}{l}
u \\
d \\
s
\end{array}\right)
$$

of the flavor $\mathrm{SU}(3)$. Since $3 \otimes 3=6 \oplus \overline{3}$ and the lowest lying light quark state has $n=1$ and $l=0$ ( $S$-wave), there are two different diquarks: a symmetric sextet $\left(s_{1}+s_{2}=1\right)$ and an antisymmetric antitriplet $\left(s_{1}+s_{2}=0\right)$. When the diquark combines with a heavy quark, the sextet contains both spin $\frac{1}{2}\left(B_{6}\right)$ and spin $\frac{3}{2}\left(B_{6}^{*}\right)$ baryons, and the antitriplet contains only spin $\frac{1}{2}\left(B_{\overline{3}}\right)$ baryons. Explicitly, the wave functions of spin $\frac{1}{2} B_{6}$ baryon are

$$
\begin{aligned}
& \left|\Sigma_{Q}^{+1} \uparrow\right\rangle=\sqrt{\frac{2}{3}}|u u\rangle|\uparrow \uparrow\rangle|Q \downarrow\rangle-\sqrt{\frac{1}{3}}|u u\rangle \sqrt{\frac{1}{2}}(|\uparrow \downarrow\rangle+|\downarrow \uparrow\rangle)|Q \uparrow\rangle, \\
& \left|\Sigma_{Q}^{0} \uparrow\right\rangle=\sqrt{\frac{2}{3}} \sqrt{\frac{1}{2}}(|u d\rangle+|d u\rangle)|\uparrow \uparrow\rangle|Q \downarrow\rangle-\sqrt{\frac{1}{3}} \sqrt{\frac{1}{2}}(|u d\rangle+|d u\rangle) \sqrt{\frac{1}{2}}(|\uparrow \downarrow\rangle+|\downarrow \uparrow\rangle)|Q \uparrow\rangle, \\
& \left.\left|\Sigma_{Q}^{-1} \uparrow\right\rangle=\left|\Sigma_{Q}^{+1} \uparrow\right\rangle_{(u \rightarrow d)}, \quad\left|\Xi_{Q}^{+1 / 2} \uparrow\right\rangle=\left|\Sigma_{Q}^{0} \uparrow\right\rangle\right\rangle_{(d \rightarrow s)}, \\
& \left|\Xi_{Q}^{\prime-1 / 2} \uparrow\right\rangle=\left|\Xi_{Q}^{\prime+1 / 2} \uparrow\right\rangle_{(u \rightarrow d)}, \quad\left|\Omega_{Q} \uparrow\right\rangle=\left|\Sigma_{Q}^{+1} \uparrow\right\rangle_{(u \rightarrow s)},
\end{aligned}
$$


where the superscript denotes the value of the isospin quantum number $I_{3}$. An asterisk on the symbol will denote a corresponding spin $\frac{2}{3}$ baryon:

$$
\begin{aligned}
& \left|\Sigma_{Q}^{*+1} \frac{1}{2}\right\rangle=\sqrt{\frac{1}{3}}|u u\rangle|\uparrow \uparrow\rangle|Q \downarrow\rangle+\sqrt{\frac{2}{3}}|u u\rangle \sqrt{\frac{1}{2}}(|\uparrow \downarrow\rangle+|\downarrow \uparrow\rangle)|Q \uparrow\rangle, \\
& \left|\Sigma_{Q}^{* 0} \frac{1}{2}\right\rangle=\sqrt{\frac{1}{3}} \sqrt{\frac{1}{2}}(|u d\rangle+|d u\rangle)|\uparrow \uparrow\rangle|Q \downarrow\rangle+\sqrt{\frac{2}{3}} \sqrt{\frac{1}{2}}(|u d\rangle+|d u\rangle) \sqrt{\frac{1}{2}}(|\uparrow \downarrow\rangle+|\downarrow \uparrow\rangle)|Q \uparrow\rangle, \\
& \left|\Sigma_{Q}^{*-1} \frac{1}{2}\right\rangle=\left|\Sigma_{Q}^{+1} \uparrow\right\rangle{ }_{(u \rightarrow d)}, \quad\left|\Xi_{Q}^{*+1 / 2} \frac{1}{2}\right\rangle=\left|\Sigma_{Q}^{0} \uparrow\right\rangle_{(d \rightarrow s)}, \\
& \left|\Xi_{Q}^{*-1 / 2} \frac{1}{2}\right\rangle=\left|\Xi_{Q}^{*+1 / 2} \frac{1}{2}\right\rangle_{(u \rightarrow d)}, \quad\left|\Omega_{Q}^{*} \frac{1}{2}\right\rangle=\left|\Sigma_{Q}^{*+1} \frac{1}{2}\right\rangle_{(u \rightarrow s)},
\end{aligned}
$$

As to the wave functions of spin $\frac{1}{2} B_{\overline{3}}$ baryon, they are

$$
\begin{aligned}
& \left|\Lambda_{Q} \uparrow\right\rangle=\sqrt{\frac{1}{2}}(|u d\rangle-|d u\rangle) \sqrt{\frac{1}{2}}(|\uparrow \downarrow\rangle-|\downarrow \uparrow\rangle)|Q \uparrow\rangle, \\
& \left|\Xi_{Q}^{+1 / 2} \uparrow\right\rangle=\left|\Lambda_{Q} \uparrow\right\rangle_{(d \rightarrow s)}, \quad\left|\Xi_{Q}^{-1 / 2} \uparrow\right\rangle=\left|\Xi_{Q}^{+1 / 2} \uparrow\right\rangle_{(u \rightarrow d)} .
\end{aligned}
$$

We may use the decomposition

$$
\begin{aligned}
q_{1 i} q_{2 j} & =\frac{1}{2}\left(q_{1 i} q_{2 j}+q_{1 j} q_{2 i}\right)+\frac{1}{2}\left(q_{1 i} q_{2 j}-q_{1 j} q_{2 i}\right) \\
& =\left(B_{6}\right)_{i j}+\frac{1}{\sqrt{2}}\left(B_{\overline{3}}\right)_{i j}
\end{aligned}
$$

to assemble the sextet and the antitriplet in a systematic and an antisymmetric $3 \times 3$ matrix, respectively,

$$
\begin{aligned}
B_{6}= & {\left[\begin{array}{ccc}
\Sigma_{Q}^{+1} & \frac{1}{\sqrt{2}} \Sigma_{Q}^{0} & \frac{1}{\sqrt{2}} \Xi_{Q}^{\prime+1 / 2} \\
\frac{1}{\sqrt{2}} \Sigma_{Q}^{0} & \Sigma_{Q}^{-1} & \frac{1}{\sqrt{2}} \Xi_{Q}^{\prime-1 / 2} \\
\frac{1}{\sqrt{2}} \Xi_{Q}^{\prime+1 / 2} & \frac{1}{\sqrt{2}} \Xi_{Q}^{\prime-1 / 2} & \Omega_{Q}
\end{array}\right], } \\
B_{\overline{3}}= & {\left[\begin{array}{ccc}
0 & \Lambda_{Q} & \Xi_{Q}^{+1 / 2} \\
-\Lambda_{Q} & 0 & \Xi_{Q}^{-1 / 2} \\
-\Xi_{Q}^{+1 / 2} & \Xi_{Q}^{-1 / 2} & 0
\end{array}\right], }
\end{aligned}
$$

and a matrix for $B_{6}^{*}$

$$
B_{6}^{*}=\left[\begin{array}{ccc}
\Sigma_{Q}^{*+1} & \frac{1}{\sqrt{2}} \Sigma_{Q}^{* 0} & \frac{1}{\sqrt{2}} \Xi_{Q}^{\prime+1 / 2} \\
\frac{1}{\sqrt{2}} \Sigma_{Q}^{* 0} & \Sigma_{Q}^{*-1} & \frac{1}{\sqrt{2}} \Xi_{Q}^{\prime *-1 / 2} \\
\frac{1}{\sqrt{2}} \Xi_{Q}^{*+1 / 2} & \frac{1}{\sqrt{2}} \Xi_{Q}^{\prime *-1 / 2} & \Omega_{Q}^{*}
\end{array}\right]
$$




\section{CHIRAL DYNAMICS OF HEAVY BARYONS}

Before discussing the interaction between heavy baryons and Goldstone bosons, we will first summarize the case of Goldstone bosons interacting among themselves [9, 10]. The chiral symmetry is nonlinearly realized by using the unitary matrix

$$
\Sigma=e^{2 i M / \sqrt{2} f_{\pi}}
$$

where $M$ is a $3 \times 3$ matrix for the octet of Goldstone bosons

$$
M=\left[\begin{array}{ccc}
\frac{\pi^{0}}{\sqrt{2}}+\frac{\eta}{\sqrt{6}} & \pi^{+} & K^{+} \\
\pi^{-} & -\frac{\pi^{0}}{\sqrt{2}}+\frac{\eta}{\sqrt{6}} & K^{0} \\
K^{-} & \bar{K}^{0} & -\sqrt{\frac{2}{3} \eta}
\end{array}\right]
$$

and $f_{\pi}=93 \mathrm{MeV}$ is the pion decay constant. Under $S U(3)_{L} \times S U(3)_{R}, \Sigma$ transforms as

$$
\Sigma \rightarrow \Sigma^{\prime}=L \Sigma R^{\dagger}
$$

Hence the lowest order Lagrangian for the Goldstone bosons is

$$
\mathcal{L}_{M}=\frac{f_{\pi}^{2}}{4} \operatorname{Tr} \partial_{\mu} \Sigma^{\dagger} \partial^{\mu} \Sigma
$$

In order to facilitate the discussions of the Goldstone bosons interacting with heavy mesons, we introduce a new Goldstone-boson matrix $\xi \equiv \Sigma^{1 / 2}$, which transforms under an $S U(3)_{L} \times$ $S U(3)_{R}$ as

$$
\xi \rightarrow \xi^{\prime}=L \xi U^{\dagger}=U \xi R^{\dagger}
$$

where $U$ is an unitary matrix depending on $L, R$, and $M$, so that it is no longer global. Now with the aid of $\xi$, we construct a vector field $V_{\mu}$ and an axial vector field $A_{\mu}$ :

$$
\begin{aligned}
V_{\mu} & =\frac{1}{2}\left(\xi^{\dagger} \partial_{\mu} \xi+\xi \partial_{\mu} \xi^{\dagger}\right) \\
A_{\mu} & =\frac{i}{2}\left(\xi^{\dagger} \partial_{\mu} \xi-\xi \partial_{\mu} \xi^{\dagger}\right)
\end{aligned}
$$

with the simple transformation properties

$$
\begin{aligned}
& V_{\mu} \rightarrow V_{\mu}^{\prime}=U V_{\mu} U^{\dagger}+U \partial_{\mu} U^{\dagger} \\
& A_{\mu} \rightarrow A_{\mu}^{\prime}=U A_{\mu} U^{\dagger}
\end{aligned}
$$


The chiral transformation of $q$ is given in Eq. (26) which is however not convenient for our purpose. By means of the following field redefinition [11]

$$
q_{L} \rightarrow \xi^{\dagger} q_{L}, \quad q_{R} \rightarrow \xi q_{R}
$$

the light quarks can be made to transform simply as

$$
q \rightarrow q^{\prime}=U q
$$

and the chiral transformations of the heavy baryons in Eqs. (22) and (23) can be established as

$$
\begin{gathered}
B_{6} \rightarrow B_{6}^{\prime}=U B_{6} U^{T} \\
B_{\overline{3}} \rightarrow B_{\overline{3}}^{\prime}=U B_{\overline{3}} U^{T} .
\end{gathered}
$$

and the covariant derivatives under chiral transformations for $B_{6}$ and $B_{\overline{3}}$ are

$$
\begin{gathered}
D_{\mu} B_{6} \equiv \partial_{\mu} B_{6}+V_{\mu} B_{6}+B_{6} V_{\mu}^{T}, \\
D_{\mu} B_{\overline{3}} \equiv \partial_{\mu} B_{\overline{3}}+V_{\mu} B_{\overline{3}}+B_{\overline{3}} V_{\mu}^{T} .
\end{gathered}
$$

A similar equations hold for $B_{6}^{*}$ and $D_{\mu} B_{6}^{*}$. Then the chiral-invariant Lagrangian is

$$
\begin{aligned}
\mathcal{L}_{B} & =\operatorname{tr}\left[\bar{B}_{6}\left(i \not D-M_{6}\right) B_{6}\right]+\frac{1}{2} \operatorname{tr}\left[\bar{B}_{\overline{3}}\left(i \not D-M_{\overline{3}}\right) B_{\overline{3}}\right] \\
& +\operatorname{tr}\left\{\bar{B}_{6}^{* \mu}\left[-g_{\mu \nu}\left(i \not D-M_{6}^{*}\right)+i\left(\gamma_{\mu} D_{\nu}+\gamma_{\nu} D_{\mu}\right)-\gamma_{\mu}\left(i \not D+M_{6}^{*}\right) \gamma_{\nu}\right] B_{6}^{* \nu}\right\} \\
& +g_{1} \operatorname{tr}\left(\bar{B}_{6} \gamma_{\mu} \gamma_{5} A^{\mu} B_{6}\right)+g_{2} \operatorname{tr}\left(\bar{B}_{6} \gamma_{\mu} \gamma_{5} A^{\mu} B_{\overline{3}}\right)+\text { H.c. } \\
& +g_{3} \operatorname{tr}\left(\bar{B}_{6 \mu}^{*} A^{\mu} B_{6}\right)+\text { H.c. }+g_{4} \operatorname{tr}\left(\bar{B}_{6 \mu}^{*} A^{\mu} B_{\overline{3}}\right)+\text { H.c. } \\
& +g_{5} \operatorname{tr}\left(\bar{B}_{6 \nu}^{*} \gamma_{\mu} \gamma_{5} A^{\mu} B_{6}^{* \nu}\right)+g_{6} \operatorname{tr}\left(\bar{B}_{\overline{3}} \gamma_{\mu} \gamma_{5} A^{\mu} B_{\overline{3}}\right)
\end{aligned}
$$

where $B_{6 \mu}^{*}$ is a Rarita-Schwinger vector-spinor field a spin $\frac{2}{3}$ particle [12] and $A_{\mu}$ is the axial field introduced in Eq. (31).

\section{APPLICATIONS}

Using the bag model wave functions constructed in the previous section, one can proceed to calculate the strong decay coupling constants $\left(g_{1} \sim g_{6}\right)$. However, the six coupling constants can be reduced to two independent ones by the heavy quark symmetry (HQS) [9]. Furthermore, these two coupling constants are independent of the heavy mass. 
Now we will begin with the model calculation for the coupling constants. The strong coupling constants describe soft pion emission. Applying PCAC, we can express a soft pion amplitude to a matrix element of the axial-vector current

$$
\left\langle B^{\prime} \pi^{a}(q) \mid B\right\rangle=\frac{q^{\mu}}{f_{\pi}}\left\langle B^{\prime}\left|A_{\mu}^{a}\right| B\right\rangle .
$$

Here we consider the strong decay $\Sigma_{Q}^{+1} \rightarrow \Lambda_{Q} \pi^{+}$firstly. The Lagrangian Eq. (40) leads to the coupling

$$
\mathcal{L}_{\Sigma_{Q} \Lambda_{Q} \pi}=\frac{g_{2}}{\sqrt{2} f_{\pi}} \bar{\Sigma}_{Q}^{+1} \gamma^{\mu} \gamma_{5} \Lambda_{Q} \partial_{\mu} \pi^{+}
$$

If we define $g_{A}^{\Sigma_{Q} \Lambda_{Q}}$ by

$$
\left\langle\Lambda_{Q}\left|A_{\mu}^{1}-i A_{\mu}^{2}\right| \Sigma_{Q}^{+1}\right\rangle=g_{A}^{\Sigma_{Q} \Lambda_{Q}}\left(q^{2}\right) \bar{u}\left(\Lambda_{Q}\right) \gamma_{\mu} \gamma_{5} u\left(\Sigma_{Q}^{+1}\right)+\cdots
$$

where the unlisted terms vanish at $q=0$. Combined Eqs. (41) and (43) and compared with Eq. (42), we obtain

$$
g_{2}=-g_{A}^{\Sigma_{Q} \Lambda_{Q}}(0)
$$

The left hand side of Eq. (43) can be calculated in the MIT bag model, which is diagrammatically illustrated in FIG. 1. The $\Delta I_{3}=-1$ transition can be described by the current $\widetilde{\Sigma}_{3}(\operatorname{choosing} \mu=3)$

$$
\widetilde{\Sigma}_{3}=\int d^{3} r\left(A_{3}^{1}-i A_{3}^{2}\right)=\int d^{3} r b_{n l m}^{\dagger d} \bar{u}_{n l m}(r, t) \gamma_{3} \gamma_{5} u_{n l m}(r, t) b_{n l m}^{u},
$$

where the superscript $u(d)$ represents $u(d)$ quark. Here the light quark states have $n=1$, $l=0$, and $m=0$ ( $S$-wave) because we deal with the $B_{6}, B_{6}^{*}$, and $B_{\overline{3}}$ baryons. Therefore we obtain

$$
g_{2}=-\mathcal{I}\left\langle\Lambda_{Q} \uparrow\left|\widetilde{\Sigma}_{3}\right| \Sigma_{Q}^{+1} \uparrow\right\rangle
$$

where

$$
\mathcal{I} \equiv 4 \pi N_{I} N_{F} \int_{0}^{R} d r r^{2}\left[j_{0}\left(X_{F} \bar{r}\right) j_{0}\left(X_{I} \bar{r}\right)-\frac{1}{3} j_{1}\left(X_{F} \bar{r}\right) j_{1}\left(X_{I} \bar{r}\right)\right],
$$

$\bar{r} \equiv r / R$, and we have used the identities

$$
\begin{aligned}
\vec{\sigma} \cdot \widehat{r} \sigma_{i} \vec{\sigma} \cdot \widehat{r} & =2 r_{i} \sigma \cdot \widehat{r}-\sigma_{i}, \\
\int d^{3} r f(r) \widehat{r}(\vec{\sigma} \cdot \widehat{r}) & =\frac{1}{3} \int d^{3} r f(r) \vec{\sigma} .
\end{aligned}
$$


Eqs. (18) and (20) then give

$$
\left\langle\Lambda_{Q} \uparrow\left|\widetilde{\Sigma}_{3}\right| \Sigma_{Q}^{+1} \uparrow\right\rangle=-\frac{1}{2} \sqrt{\frac{1}{6}}(-4)\left\langle d \uparrow\left|\widetilde{\Sigma}_{3}\right| u \uparrow\right\rangle=\sqrt{\frac{2}{3}}
$$

The decay width of the decay $\Sigma_{Q}^{+1} \rightarrow \Lambda_{Q} \pi$ is

$$
\Gamma\left(\Sigma_{Q} \rightarrow \Lambda_{Q} \pi\right)=\frac{|\vec{p}|}{8 \pi M_{\Sigma_{Q}}^{2}} g_{\Sigma_{Q} \Lambda_{Q} \pi}^{2}\left[\left(M_{\Sigma_{Q}}-M_{\Lambda_{Q}}\right)^{2}-M_{\pi}^{2}\right]
$$

where $\vec{p}$ is the pion momentum in the c.m. system and

$$
g_{\Sigma_{Q} \Lambda_{Q} \pi}=\frac{M_{\Sigma_{Q}}+M_{\Lambda_{Q}}}{\sqrt{2} f_{\pi}} g_{2}
$$

is the Goldberger-Treiman relation.

Next, we consider the strong decay $\Sigma_{Q}^{*+1} \rightarrow \Lambda_{Q} \pi$. The Lagrangian Eq. (40) leads to the coupling

$$
\mathcal{L}_{\Sigma_{Q}^{*} \Lambda_{Q} \pi}=\frac{g_{4}}{\sqrt{2} f_{\pi}} \bar{\Sigma}_{Q \mu}^{*+1} \Lambda_{Q} \partial^{\mu} \pi^{+}
$$

We may take the advantage of the constraints imposed by HQS [9]

$$
g_{4}=-\sqrt{3} g_{2}
$$

The decay width of the decay $\Sigma_{Q}^{*+1} \rightarrow \Lambda_{Q} \pi$ is

$$
\Gamma\left(\Sigma_{Q}^{*} \rightarrow \Lambda_{Q} \pi\right)=\frac{|\vec{p}|^{3}}{96 \pi M_{\Sigma_{Q}^{*}}^{2} M_{\Lambda_{Q}}^{2}} g_{\Sigma_{Q}^{*} \Lambda_{Q} \pi}^{2}\left[\left(M_{\Sigma_{Q}^{*}}+M_{\Lambda_{Q}}\right)^{2}-M_{\pi}^{2}\right]
$$

where

$$
g_{\Sigma_{Q}^{*} \Lambda_{Q} \pi}=\frac{M_{\Sigma_{Q}^{*}}+M_{\Lambda_{Q}}}{\sqrt{2} f_{\pi}} g_{4}
$$

is also the Goldberger-Treiman relation.

The parameters we use as input to calculate $\mathcal{I}$ are the light quark masses $m_{u, d}$ and the bag of radius $R$. The current light quark masses in general are taken as $m_{u, d}=0$. As to the radius $R, R_{\Sigma_{c}^{++}}=0.945 \mathrm{fm}, R_{\Sigma_{c}^{*++}}=1.01 \mathrm{fm}$, and $R_{\Lambda_{c}^{+}}=0.914 \mathrm{fm}$ were given by Ref. [13] in charm sector, and $R_{\Sigma_{b}^{+}}=1.02 \mathrm{fm}, R_{\Sigma_{b}^{*+}}=1.04 \mathrm{fm}$, and $R_{\Lambda_{b}^{0}}=0.996 \mathrm{fm}$ were given by Ref. [14] in bottom sector. They were obtained by fitting the mass spectrum in the respective sectors. Here we use the parameter $m_{u, d}=0$ and $R=1 \mathrm{fm}$ to Eq. (47) and obtain $\mathcal{I}=0.653$. Then the strong decay widths in Eqs. (49) and (53) can be estimated, and the 


\begin{tabular}{c|c|c|c}
\hline \hline & $\Gamma\left(\Sigma_{c}^{++} \rightarrow \Lambda_{c}^{+} \pi^{+}\right)$ & $\Gamma\left(\Sigma_{c}^{+} \rightarrow \Lambda_{c}^{+} \pi^{0}\right)$ & $\Gamma\left(\Sigma_{c}^{0} \rightarrow \Lambda_{c}^{+} \pi^{-}\right)$ \\
\hline experiment [2] & $2.23 \pm 0.30$ & $<4.6(\mathrm{CL}=90 \%)$ & $2.2 \pm 0.4$ \\
this work & 1.90 & 2.20 & 1.87 \\
CQM [15] & $1.31 \pm 0.04$ & $1.31 \pm 0.04$ & $1.31 \pm 0.04$ \\
CQM [16] & $2.025_{-0.987}^{+1.134}$ & & $1.939_{-0.954}^{+1.114}$ \\
HHCPT [9] & $2.47,4.38$ & $2.85,5.06$ & $2.45,4.35$ \\
HHCPT [17] & 2.5 & 3.2 & 2.4 \\
HHCPT [18] & & & $1.94 \pm 0.57$ \\
HHCPT [19] & input & $2.6 \pm 0.4$ & $2.2 \pm 0.3$ \\
LFQM [20] & 1.64 & 1.70 & 1.57 \\
RTQM [21] & $2.85 \pm 0.19$ & $3.63 \pm 0.27$ & $2.65 \pm 0.19$ \\
NRQM [22] & $2.41 \pm 0.07 \pm 0.02$ & $2.79 \pm 0.08 \pm 0.02$ & $2.37 \pm 0.07 \pm 0.02$ \\
\hline \hline
\end{tabular}

TABLE II: Decay widths $\Gamma\left(\Sigma_{c} \rightarrow \Lambda_{c} \pi\right)$ (in units of MeV). Experimental data and other theoretical calculations are also shown.

numerical results in charm sector are listed in TABLE II and TABLE III, respectively. We find that the results are all consistent with the experimental data. In addition, if the value of $m_{u, d}$ varies from 0 to $8 \mathrm{MeV}$ and $R$ varies from $0.8 \mathrm{fm}$ to $1.2 \mathrm{fm}$, then the decay width, for example, $\Gamma\left(\Sigma_{c}^{++} \rightarrow \Lambda_{c}^{+} \pi^{+}\right)$varies just from 1.90 to $1.94 \mathrm{MeV}$. That is, these calculations are insensitive to values of $m_{u, d}$ and $R$. We also present the experimental data and other theoretical calculations in the tables.

Finally, we use the same parameters to predict the relevant strong decay widths in bottom sector. Because the neutral bottomed baryons $\Sigma_{b}^{0(*)}$ have not found yet, we resort to the following supposition to obtain $M_{\Sigma_{b}^{(*) 0}}$ : the mass differences $\Delta M_{b}$ among bottomed baryons $\Sigma_{b}^{+}, \Sigma_{b}^{0}$, and $\Sigma_{b}^{-}$come from two parts, one part is the quark mass difference $m_{d}-m_{u}$, and the other part is the electromagnetic Coulomb energy. Thus we may take the quark replacement $b \rightarrow s$ and observe the mass differences among $\Sigma^{+}, \Sigma^{0}$, and $\Sigma^{-}$. From Ref. [2], it is easily to find that

$$
M_{\Sigma^{0}} \simeq \frac{1}{2}\left(M_{\Sigma^{+}}+M_{\Sigma^{-}}\right), \quad M_{\Sigma^{* 0}} \simeq \frac{1}{2}\left(M_{\Sigma^{*+}}+M_{\Sigma^{*-}}\right)
$$

The errors are both smaller than $10^{-3}$. Therefore, we may approximately use the similar 


\begin{tabular}{c|c|c|c}
\hline \hline & $\Gamma\left(\Sigma_{c}^{*++} \rightarrow \Lambda_{c}^{+} \pi^{+}\right)$ & $\Gamma\left(\Sigma_{c}^{*+} \rightarrow \Lambda_{c}^{+} \pi^{0}\right)$ & $\Gamma\left(\Sigma_{c}^{* 0} \rightarrow \Lambda_{c}^{+} \pi^{-}\right)$ \\
\hline experiment [2] & $14.9 \pm 1.9$ & $<17(\mathrm{CL}=90 \%)$ & $16.1 \pm 2.1$ \\
this work & 14.7 & 15.2 & 14.5 \\
CQM [15] & 20 & 20 & 20 \\
HHCPT [17] & 25 & 25 & 25 \\
HHCPT [19] & $16.7 \pm 2.3$ & $17.4 \pm 2.3$ & $16.6 \pm 2.2$ \\
LFQM [20] & 12.84 & & 12.40 \\
RTQM [21] & $21.99 \pm 0.87$ & & $21.21 \pm 0.81$ \\
NRQM [22] & $17.52 \pm 0.74 \pm 0.12$ & $17.31 \pm 0.73 \pm 0.12$ & $16.90 \pm 0.71 \pm 0.12$ \\
\hline \hline
\end{tabular}

TABLE III: Decay widths $\Gamma\left(\Sigma_{c}^{*} \rightarrow \Lambda_{c} \pi\right)$ (in units of MeV). Experimental data and other theoretical calculations are also shown.

equations

$$
M_{\Sigma_{b}^{0}} \rightarrow \frac{1}{2}\left(M_{\Sigma_{b}^{+}}+M_{\Sigma_{b}^{-}}\right), \quad M_{\Sigma_{b}^{* 0}} \rightarrow \frac{1}{2}\left(M_{\Sigma_{b}^{*+}}+M_{\Sigma_{b}^{*-}}\right)
$$

The results of the decay widths $\Gamma\left(\Sigma_{b}^{(*)} \rightarrow \Lambda_{b} \pi\right)$ are listed in TABLE IV.

\begin{tabular}{c|c|c||c|c|c}
\hline \hline$\Gamma\left(\Sigma_{b}^{+} \rightarrow \Lambda_{b}^{0} \pi^{+}\right)$ & $\Gamma\left(\Sigma_{b}^{0} \rightarrow \Lambda_{b}^{0} \pi^{0}\right)$ & $\Gamma\left(\Sigma_{b}^{-} \rightarrow \Lambda_{b}^{0} \pi^{-}\right)$ & $\Gamma\left(\Sigma_{b}^{*+} \rightarrow \Lambda_{b}^{0} \pi^{+}\right)$ & $\Gamma\left(\Sigma_{b}^{* 0} \rightarrow \Lambda_{b}^{0} \pi^{0}\right)$ & $\Gamma\left(\Sigma_{b}^{*-} \rightarrow \Lambda_{b}^{0} \pi^{-}\right)$ \\
\hline 4.35 & 5.65 & 5.77 & 8.50 & 10.20 & 10.44 \\
\hline \hline
\end{tabular}

TABLE IV: The predictions of the strong decay widths $\Gamma\left(\Sigma_{b}^{(*)} \rightarrow \Lambda_{b} \pi\right)$ (in units of MeV).

\section{CONCLUSION}

In this paper we have presented a formalism to describe the chiral dynamics of the heavy baryons interacting with the Goldstone bosons. Furthermore, through PCAC, we obtained the relevant strong constants in the MIT bag model, and then estimated the strong decay widths $\Gamma\left(\Sigma_{b, c}^{(*)} \rightarrow \Lambda_{b, c} \pi\right)$. The parameters appearing in this approach are the light quark masses $m_{u, d}$ and the bag radius $R$. On the one hand the current masses of $u, d$ quarks are both very small on the typical hadron energy scale and can be taken as zero, and on the 
other hand the bag radius $R=1 \mathrm{fm}$ was obtained by fitting the mass spectrum in the charm

[13] and bottom [14] sectors and averaging them. In addition, we also separately vary $m_{u, d}$ from 0 to $8 \mathrm{MeV}$ and $R$ from 0.8 to $1.2 \mathrm{fm}$, and find the decay widths are insensitive to these two parameters. Base on the numerical results being all consistent with the experimental data in charm sector, we make the predictions on the strong decay widths $\Gamma\left(\Sigma_{b}^{(*)} \rightarrow \Lambda_{b} \pi\right)$. In spite that the bottomed baryons $\Sigma_{b}^{(*) 0}$ have not been found, we observed that the masses of neutral baryons $M_{\Sigma^{(*) 0}}$ almost equal to the average of the ones of charged baryons $M_{\Sigma^{(*)+}}$ and $M_{\Sigma^{(*)-}}$, and then analogized to the masses of neutral bottomed baryons $M_{\Sigma_{b}^{(*) 0}}$. We expect the deviations of this assumption and the predictive strong decay widths are all small for the future experimental data.

\section{Acknowledgments}

I would like to thank Dr. Chun-Khiang Chua for helpful discussion. This work is supported in part by the National Science Council of R.O.C. under Grant No: NSC-95-2112-M-017-001.

[1] Igor V. Gorelov (CDF Collaboration), hep-ex/0701056.

[2] W.-M. Yao et al., J. of Phys. G 33, 1 (2006).

[3] A. Chodos, R. L. Jaffe, K. Johnson, C. B. Thorn, and V. F. Weisskopf, Phys. Rev. D 9, 3471 (1974).

[4] A. Chodos, R. L. Jaffe, K. Johnson, and C. B. Thorn, Phys. Rev. D 10, 2599 (1974).

[5] T. DeGrand, R. L. Jaffe, K. Johnson, and J. Kiskis, Phys. Rev. D 12, 2060 (1975).

[6] D. Izatt and C. Detar, Nucl. Phys. B 199, 269 (1982).

[7] P. N. Bogolioubov, Ann. Inst. Henri Poincare 8, 163 (1967).

[8] T. Barnes, Phys. Rev. D30, 1961 (1984).

[9] T. M. Yan, H. Y. Cheng, C. Y. Cheung, G. L. Lin, Y. C. Lin, and H. L. Yu, Phys. Rev. D 46, 1148 (1992); D $\underline{\mathbf{5 5}}, 5851 \mathrm{E}(1997)$.

[10] H. Georgi, Weak Interactions and Modern Particle Theory (Benjamin, New York, 1984).

[11] C. Y. Cheung, Chinese J. Phys. 32, 1221 (1994).

[12] W. Rartia and J. Schwinger, Phys. Rev. 60, 61 (1941).

[13] S. K. Bose and L. P. Singh, Phys. Rev. D 22, 773 (1980).

[14] W. A. Ponce, Phys. Rev. D 19, 2197 (1979). 
[15] J. L. Rosner, Phys. Rev. D 52, 6461 (1995).

[16] D. Pirjol and T. M. Yan, Phys. Rev. D 56, 5483 (1997).

[17] M.-Q. Huang, Y.-B. Dai, and C.-S. Huang, Phys. Rev. D 52, 3986 (1995).

[18] H.-Y. Cheng, Phys. Lett. B 399, 281 (1997).

[19] H. Y. Cheng and C. K. Cuha, Phys. Rev. D 75, 014006 (2007).

[20] S. Tawfiq, P. J. O’Donnell, and J. G. Körner, Phys. Rev. D 58, 054010 (1997).

[21] M. A. Ivanov, J. G. Körner, V. E. Lyubovitskij, and A. G. Rusetsky, Phys. Rev. D 60, 094002 (1999); Phys. Lett. B 442, 435 (1998).

[22] C. Albertus, E. Hernández, J. Nieves, and J. M. Verde-Velasco, Phys. Rev. D 72, 094022 (2005). 


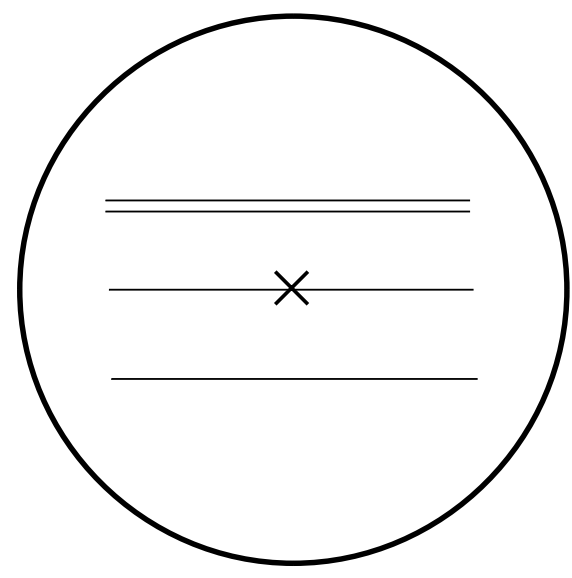

FIG. 1: Single particle transition in the bag. The single (double) line stands for a light (heavy) quark, and the cross represents an external current operator. 